\title{
The solar chromosphere as seen in high-resolution millimeter observations
}

\author{
M.A. Loukitcheva ${ }^{1,2}$, S.K. Solanki ${ }^{2}$ \\ and S. White ${ }^{3}$ \\ ${ }^{1}$ Astronomical Institute, Saint-Petersburg State University, St. Petersburg, Russia, email: \\ marija@peterlink.ru \\ 2 Max-Planck-Institut für Sonnensystemforschung, Katlenburg-Lindau, Germany \\ ${ }^{3}$ Astronomy Department, University of Maryland, College Park, USA
}

\begin{abstract}
We report on chromospheric oscillations studied with $15 \mathrm{sec}$ cadence observations obtained at a wavelength of $3.5 \mathrm{~mm}$ with the Berkeley-Illinois-Maryland Array (BIMA). Different solar structures, including active and quiet-Sun regions, are analyzed. We compare the high-resolution millimeter 2-D images with Ca II K line images from Big Bear Solar Observatory. Using Fourier and wavelet time series analysis techniques we find signatures of intensity oscillations with periods in the range of 2-7 minutes. We discuss the results in the context of the dynamical model of the solar atmosphere by Carlsson \& Stein $(1995,1997)$.
\end{abstract}

\section{Observations and data analysis}

In this contribution we present observations of chromospheric oscillations at $3.5 \mathrm{~mm}$ and compare them with the intensity variations expected from the Carlsson \& Stein (1995, 1997) model (CS), which have been computed for wavelengths in the millimeter range of $0.5-20 \mathrm{~mm}$ by Loukitcheva et al. (2004).

The chromospheric radio data were obtained on August 31, 2003 with the BIMA interferometer consisting of ten $6.1 \mathrm{~m}$ diameter dishes. The deconvolved images were restored with a Gaussian beam of 10 arcsec and corrected for the primary beam size. The estimated noise level was typically about $100 \mathrm{~K}$ in brightness temperature.

In order to provide a context for understanding the millimeter observations, we compared them with the Ca II K line images cotemporally recorded at the Big Bear Solar Observatory (BBSO). Both active region (AR) and quiet Sun (QS) images in $85 \mathrm{GHz}$ emission show a very good correlation with the Ca II $\mathrm{K}$ line images, which raises our confidence in the BIMA images.

We have performed tests of the sensitivity of millimeter interferometer data to oscillations and concluded that oscillating sources can be recovered down to quite low amplitudes (less than $100 \mathrm{~K}$ ), which indicates that the data are suitable to look for oscillation power.

Oscillation power in the millimeter images: We have calculated power spectra for each point within the BIMA primary beam. For two frequency domains of interest, 3 min and $5 \mathrm{~min}$, frequency binning was carried out and two-dimensional power maps were constructed for the quiet sun region and active region. Power is almost always present at multiple periods, but there is more power in the 5-minute maps than in the 3 -minute maps for both the QS and AR targets. The spatial distribution of power follows the brightness distribution and 5-minute power favors higher brightness values. For an investigation of magnetic and non-magnetic behavior of the quiet Sun at millimeter wavelengths and comparison with the results from other observations each observed field was divided into 
internetwork (IN), network (NW) and remaining (intermediate) area based on masks constructed from the BBSO Ca II K images.

Comparison with the CS model: The evolution of the millimeter spectrum with time was studied on the basis of the CS dynamic model by Loukitcheva et al. (2004). In this work we compare the oscillations predicted by the model with the observed variations for the quiet Sun. Due to limited observational spatial resolution (10 arcsec) and the unknown oscillation coherence length we are not able to compare observations and model directly. We model the effect of limited spatial resolution by mixing model time series with random phase shifts. For comparison with NW regions we have employed adding of a sinusoid component corresponding to longer network periods.

\section{Results and Discussion}

Our work demonstrates that high resolution millimeter wave observations are a very promising diagnostic of chromospheric structure and dynamics. Intensity oscillations with significant power at periods in the range of 2-7 minutes were found in QS and AR structures. The spatially resolved observations show the presence of short period oscillations in the internetwork and longer periods in the network in good agreement with results obtained at other wavelengths. This is to our knowledge the first detection of spatially resolved chromospheric oscillations at millimeter wavelengths. A direct comparison of model radio brightness variations with the observations reveals a much larger variability in the Carlsson and Stein model than in the observations ( $800 \mathrm{~K}$ vs. $130 \mathrm{~K} \mathrm{rms}$ ). However, if we model the influence of seeing by mixing different oscillations with random phases, a remarkably similar signature is shown by both observations and model. Unfortunately, at present it cannot be judged if the amount of spatial smearing we employ is appropriate or not. Observations with still higher resolution are needed to settle the nature of the solar chromosphere.

\section{Acknowledgements}

The use of BIMA for scientific research carried out at the University of Maryland is supported by NSF grant AST-0028963. Solar research at the University of Maryland is supported by NSF grant ATM 99-90809 and NASA grants NAG 5-8192, NAG 5-10175, NAG 5-12860 and NAG 5-11872. Part of the work was supported by INTAS (grant 00-543) and Russian Federal Program (project 40.022.1.1.1105).

\section{References}

Carlsson, M. \& Stein, R.F. 1995, ApJ, 440, L29-L32.

Carlsson, M. \& Stein, R.F. 1997, ApJ, 481, 500-514.

Loukitcheva, M., Solanki, S.K., Carlsson, M., Stein, R.F. 2004, A\&A, 419, 747-756. 